\section{Response to: 'Evaluation of the association between anticarbamylated protein antibodies and longitudinal course of functional ability in rheumatoid arthritis' by Ajeganova et al}

We thank Ajeganova et $a l^{1}$ for the interesting comments on our paper ${ }^{2}$, and note the different findings compared with our results. ${ }^{1} 2$ We would suggest a potential explanation for this lies within the different patient populations. Although inclusion criteria for the Swedish Better Anti-Rheumatic PharmacOTherapy (BARFOT) cohort and Leiden Early arthritis Clinic (EAC) are not detailed, we are aware from previous publications that inclusion criteria to these cohorts require patients to satisfy the 1987 American College of Rheumatology (ACR) classification criteria for rheumatoid arthritis (RA) at baseline and within 1 year of symptom onset, respectively. ${ }^{3}{ }^{4}$ Our study included all patients recruited to the Norfolk Arthritis Register (NOAR) cohort from 1990-1994 and 2000-2009, where the only inclusion criteria are $\geq$ two swollen joints for $\geq 4$ weeks. The NOAR cohort therefore includes a much broader group of patients with inflammatory arthritis. It is well recognised that not all patients with persistent inflammatory arthritis are identified by RA classification criteria, particularly the 1987 criteria in early arthritis. In a sensitivity analysis on the subset of patients in NOAR who satisfied the 1987 criteria at baseline we found that the association between anticarbamylated protein (anti-CarP) antibodies and Health Assessment Questionnaire (HAQ) scores over time was smaller (adjusted $\beta$ coefficient (95\% CIs) was $0.09(-0.04$ to 0.23$)$ ), and we reported similar results in the supplementary data file accompanying our manuscript on patients who satisfy the $2010 \mathrm{ACR} /$ European League Against Rheumatism classification criteria for RA. ${ }^{2}$ These results are more in keeping with those found in the BARFOT and EAC. They suggest that the specific utility of testing for anti-CarP antibodies may lie in patients who may not satisfy RA classification criteria early on and therefore have been excluded from the BARFOT or EAC studies, but are found in NOAR. We contend this argues in favour of testing for these antibodies; they provide information and predict outcomes in a group of patients that we know little about as they are often excluded from cohorts at the outset.

It should also be noted that the duration of follow-up within the BARFOT and EAC patients reported in their analysis is a maximum of 5 years in EAC and 8 years in BARFOT, whereas in our data there were up to 20 years' follow-up data. The statistical methods also differ. Ajeganova et al plotted mean values at each time point, while we plotted the Generalised Estimating Equations (GEE) modelled data. The variation in statistical techniques is likely to only have marginally affected the results, however in combinations with the differences between the patient cohorts they may have contributed to the contrasting results seen in NOAR versus the other two cohorts.

We believe that we demonstrated that anti-CarP antibodies represent an important new biomarker for predicting long-term outcome in patients with early inflammatory arthritis. It is of interest that they may provide less additional prognostic information in patients with definite rheumatoid arthritis and a higher prevalence of other autoantibodies.

\section{J H Humphreys, ${ }^{1}$ D P M Symmons, ${ }^{1,2}$ S M M Verstappen ${ }^{1}$}

${ }^{1}$ Arthritis Research UK Centre for Epidemiology, University of Manchester, Manchester, UK

${ }^{2}$ NIHR Manchester Musculoskeletal Biomedical Research Unit, Manchester Academic Health Science Centre, Manchester, UK

Correspondence to Dr Suzanne Verstappen, Arthritis Research UK Centre for Epidemiology, University of Manchester, Manchester, M13 9PT, UK; suzanne. verstappen@manchester.ac.uk

Competing interests None declared.

Provenance and peer review Commissioned; internally peer reviewed.

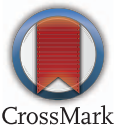

To cite Humphreys JH, Symmons DPM, Verstappen SMM. Ann Rheum Dis 2016;75:e15.

Received 15 January 2016

Accepted 16 January 2016

\section{SLinked}

- http://dx.doi.org/10.1136/annrheumdis-2015-208954

Ann Rheum Dis 2016:75:e15. doi:10.1136/annrheumdis-2015-208957

REFERENCES

1 Ajeganova S, Svensson B, Huizinga TW, et al. Evaluation of the association between anti-carbamylated protein antibodies and the longitudinal course of functional ability in rheumatoid arthritis. Ann Rheum Dis 2016;75:e14.

2 Humphreys JH, Verheul MK, Barton A, et al. Anticarbamylated protein antibodies are associated with long-term disability and increased disease activity in patients with early inflammatory arthritis: results from the Norfolk Arthritis Register. Ann Rheum Dis 2015; Published Online First 6 Oct 2015. http://dx.doi.org/10.1136/annrheumdis2015-207326

3 de Rooy DP, van der Linden MP, Knevel R, et al. Predicting arthritis outcomes-what can be learned from the Leiden Early Arthritis Clinic? Rheumatology (Oxford) 2011; 50:93-100.

4 Svensson B, Boonen A, Albertsson $\mathrm{K}$, et al. Low-dose prednisolone in addition to the initial disease-modifying antirheumatic drug in patients with early active rheumatoid arthritis reduces joint destruction and increases the remission rate: a two-year randomized trial. Arthritis Rheum 2005:52:3360-70. 